\title{
A New Robust Multi-station TDOA Localization Algorithm
}

\author{
Jianghuai Pan* \\ Jiangsu Automation Research Institute, Lianyungang 222006 China \\ ${ }^{*}$ Corresponding author
}

\begin{abstract}
During the multi-station TDOA Localization, instable or divergent positioning evaluation result, even invalid situation will occur caused by unreasonable sensor allocation, and analyzes the causes of ill-condition in traditional TDOA localization algorithm Therefore, this paper puts forward a kind of stable positioning method based on singular value decomposition modification. The method doesn't require the station allocation greatly and could consider the resolution and variance assessed by positioning parameter effectively. It realizes effective inhibition to the random observation noise in the observation equation and has better engineering application value. The value simulation result indicates the positioning result is more precise and stable than traditional calculating method.
\end{abstract}

Keywords- TDOA; passive localization; Modified Singular Value Decomposition(MSVD); robust estimation; localization algorithm

\section{INTRODUCTION}

In modern war, passive localization can locate the target radiated by the passive electromagnetic wave. It has a strong concealment and is becoming more and more important in electronic warfare. At present all kinds of electronic warfare systems, electronic reconnaissance equipment generally has a measurement of the TDOA(Time Difference of Arrive), one of the most common parameters of target parameters also can obtain the direction of the station ${ }^{[1,2]}$, multi TDOA localization algorithm is the earliest and the technology is relatively mature passive positioning method ${ }^{[3,4]}$. The domestic and foreign scholars in the multi station TDOA location precision analysis, optimal station analysis, tracking and elimination of false location has done a lot of work and achieved certain results, but compared with other methods ${ }^{[5,6]}$, the multi station TDOA location precision of direction finding error influence, in the localization algorithm ${ }^{[7]}$, optimal station analysis, tracking filter and so there is room for further improvement ${ }^{[8,9]}$.

The multi station TDOA algorithm for observability analysis, because the data is less or unreasonable layout of observability resulting in lower case, proposes a modified robust singular value decomposition method based on multi station cross location. The method of the station layout requirements is not high, can effectively combine positioning parameter estimation resolution and variance, to achieve effective suppression of the random observation noise observation equation, which improves the positioning accuracy and stability of estimation of the validity of the simulation results proves that the algorithm.

\section{MUlti StATION TdOA LOCATION MODEL}

The multi station TDOA location requires three pairs of hyperboloid formed by three baselines to locate the emitter in three dimensions, this requires that at least four stations simultaneously complete the measurement of the time difference of the arrival time of the target signal. For the signal of the target radiation, three relative delay differences can be obtained at the four stations, define a master receiving station and measure the delay difference with one of the secondary stations, in two the main line station receiving station and receiving station as the axis can be a rotating hyperboloid, two rotating hyperboloid intersection get an intersection, the intersection and third hyperboloid intersection, an intersection location, this intersection is the target location. and the positioning map is shown in figure 1 .

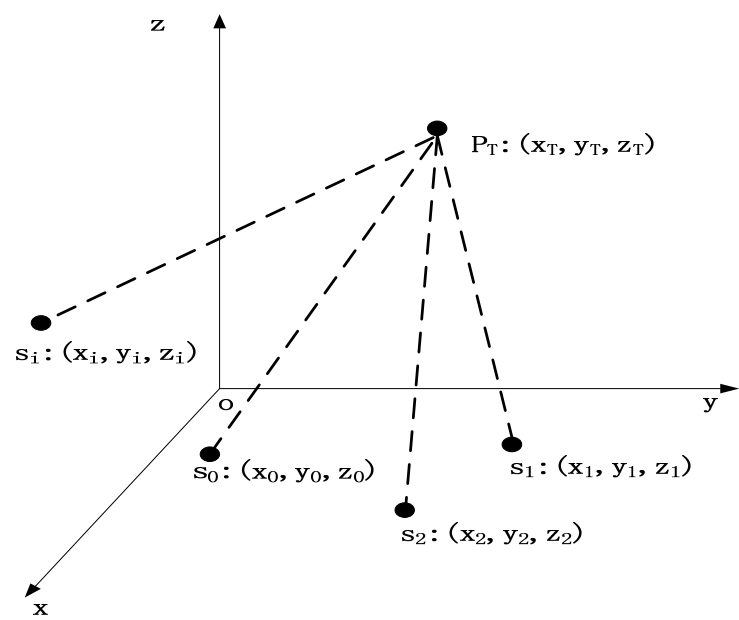

FIGURE I. FIGURE. OF MULTI STATION TDOA LOCATION IN THREE-DIMENSIONAL SPACE

Suppose the station $\mathrm{s}_{0}$ is the master station, that is, when it is $i=0$, it stands for the master station, $i=1, \ldots, n$ stands for the secondary station, $\mathrm{r}_{\mathrm{i}}(\mathrm{i}=0,1, \ldots, \mathrm{n})$ represents the spatial distance from the target to the ith station, and $\Delta r_{i}(\mathrm{i}=0,1, \ldots, \mathrm{n})$ represents the distance difference between the target and the master station, so we can have the following difference equation: 


$$
\begin{gathered}
\left\{\begin{array}{l}
r_{0}^{2}=\left(x-x_{0}\right)^{2}+\left(y-y_{0}\right)^{2}+\left(z-z_{0}\right)^{2} \\
r_{i}^{2}=\left(x-x_{i}\right)^{2}+\left(y-y_{i}\right)^{2}+\left(z-z_{i}\right)^{2}
\end{array}\right. \\
\Delta r_{i}=r_{i}-r_{0}=c \cdot \Delta t_{i} ; i=1,2, \cdots, n
\end{gathered}
$$

Where: $r_{0}$ is the target to master the distance, $r_{i}$ is the target to the auxiliary station distance, assuming the target position $(x, y, z),\left(x_{0}, y_{0}, z_{0}\right)$ is the master coordinate sensor in geographic coordinate system, $\left(x_{i}, y_{i}, z_{i}\right)$ is the $i$ th coordinates of sensors in the geographic coordinate system, $\Delta r_{i}$ is the source to the main station to the secondary station and the distance between the poor, $\Delta t_{i}$ is the radiation source signal to the master and auxiliary to the time difference between the stations , $c$ is the speed of signal propagation..

The development of equations (1) can be simplified

$$
\left(x_{0}-x_{i}\right) x+\left(y_{0}-y_{i}\right) y+\left(z_{0}-z_{i}\right) z=k_{i}+r_{0} \Delta r_{i}
$$

Where: $k_{i}=\frac{1}{2}\left(\Delta_{i}^{2}+\left(x_{0}^{2}+y_{0}^{2}+z_{0}^{2}\right)-\left(x_{i}^{2}+y_{i}^{2}+z_{i}^{2}\right)\right), i=1,2, \cdots ; n$.

Write all sensor observations(2) in matrix form.

$$
A X=Z
$$

Where: $A=\left[\begin{array}{ccc}x_{0}-x_{1} & y_{0}-y_{1} & z_{0}-z_{1} \\ x_{0}-x_{2} & y_{0}-y_{2} & z_{0}-z_{2} \\ \vdots & \vdots & \vdots \\ x_{0}-x_{n} & y_{0}-y_{n} & z_{0}-z_{n}\end{array}\right], X=\left[\begin{array}{c}x \\ y \\ z\end{array}\right], Z=\left[\begin{array}{c}k_{1}+\Delta_{1} \cdot r_{0} \\ k_{2}+\Delta_{2} \cdot r_{0} \\ \vdots \\ k_{n}+\Delta_{n} \cdot r_{0}\end{array}\right]$.

The least square solution of the formula (3) is:

$$
\hat{X}=\left(A^{T} A\right)^{-1} A^{T} Z
$$

Defines the target localization parameter $X$ as the information matrix:

$$
J=A^{T} A
$$

\section{ILL-CONDITION ANALYSIS OF TARGET LOCATION PARAMETER ESTIMATION}

In the solution process of formula (3), the condition of the stable solution is that the information matrix $\mathrm{J}$ is invertible, that is, the $A$ column is full rank. In practical calculation, the information matrix $J$ is ill conditioned when the linear or weak correlation of the column of the matrix $A$ is caused due to the unreasonable station or the observation noise, due to the influence of random error, the solution is extremely unstable, and the error of localization is very large. From the point of view of numerical solution, formula (3) is ill conditioned, and the accuracy of direct solution formula (4) is very poor. In the common ill conditioned scenes of cross location: when the positions of sensors are closer, that is $\left(x-x_{1}, y-y_{1}, z-z_{1}\right) \approx\left(x-x_{i}, y-y_{i}, z-z_{i}\right)$, the column vectors of matrix $A$ are correlated, which leads to the existence of ill-conditioned information matrix $J$.

In order to effectively evaluate the degree of ill-conditioned, it is necessary to give an index to evaluate it quantitatively. In general, the condition number is evaluated by a number of illcondition. For the formula(5), small disturbance caused by the estimation of the relative relation of the system error are as follows:

$$
\frac{\|\delta X\|}{\|X\|} \lesssim \operatorname{cond}\{A\}\left(\frac{\|\delta A\|}{\|A\|}+\frac{\|\delta Z\|}{\|Z\|}\right)
$$

When the matrix $A$ is nonsingular, the definition of the condition number as follows:

$$
\text { cond }\{A\}=\max _{i}\left|\lambda_{i}(A)\right| / \min _{i}\left|\lambda_{i}(A)\right|
$$

The coefficient matrix of the value of $A$ is more dispersed, the bigger the cond $\{\mathrm{A}\}$, the solution vector relative error $\mathrm{B}$ is higher. Therefore, to reduce the relative error of the solution vector should be considered to reduce the $\operatorname{cond}\{\mathrm{A}\}$. The condition number is a relative number, and the degree of the minimum characteristic root is the smallest, and the condition number is a measure of the severity of the ill condition. However, there are some relations between the conditions of observation matrix, and it can't be sure that each correlation exists between them. This information has important reference value for us to grasp the mechanism and essence of the ill posed nature, and to determine which of the bias parameters.

Define the condition index:

$$
\eta_{k}=\frac{\max _{j}\left|\lambda_{j}\right|}{\left|\lambda_{k}\right|}
$$

$\eta_{k}$ define as the matrix of the $\mathrm{k}$ condition index, $\lambda_{1}$ is the maximum eigenvalues, obviously $\eta_{k} \geq 1$.

The condition index directly reflects the specific system error parameters estimation of influence degree of the measurement noise. If there is high condition index, the column element matrix perturbation, the estimation error of the parameters of the system will cause a considerable change, that there is a relationship between the data column of the observation matrix. Extensive simulation studies show that (Belsley, 1991; Davey, 1959) ${ }^{[12]}$, if the sick is very weak, condition index is less than 100; pathological condition index is 
strong, between 100 to 1000 ; the pathological condition is very serious, the index above 1000 .

\section{Robust Estimation Algorithm fOR MUlti STATION DIRECTION FINDING CROSS LOCATION}

The LS estimator of formula (3) is the best linear unbiased estimator with the least variance, but in the presence of ill conditioned parameter estimation, the estimation quality of formula (4) becomes worse, and even the results are unreliable, The larger variance makes the formula (4) become a de biased estimator in fact. When the observation equations are ill conditioned, we can be corrected by the rank of the observation matrix to obtain more robust estimation results.

By the Fisher information matrix definition that $J$ is a real symmetric matrix, according to the singular value decomposition theorem for arbitrary symmetric matrix $J_{m \times m}$, the existence of orthogonal matrix $V_{m \times m}$ :

$$
J=V S V^{T}
$$

Where $S=\left[\begin{array}{ll}\Sigma & 0 \\ 0 & 0\end{array}\right], \quad \sum=\operatorname{diag}\left(\lambda_{1}, \lambda_{2}, \ldots, \lambda_{r}\right)$, $\lambda_{1} \geq \lambda_{2} \geq \ldots \geq \lambda_{r}>0 \quad, \quad \lambda_{i} \quad$ is $J$ eigenvalues ; $r \leq \min (m, n)$. Matrix $V$ can be expressed as a column vector of matrix $V=\left(v_{1}, v_{2}, \ldots, v_{n}\right)$.

The $J$ Mooer-Penrose generalized inverse is:

$$
J^{+}=V S^{-1} V^{T}
$$

Where $S^{-1}=\operatorname{diag}\left(\lambda_{1}^{-1}, \lambda_{2}^{-1}, \ldots, \lambda_{r}^{-1}, 0, \ldots, 0\right)$

The bias estimation of variance:

$$
\mathrm{D}(\hat{b})=\sigma_{0}^{2} V S^{-2} V^{T}
$$

Written in vector form:

$$
\operatorname{var}(\hat{b})=\sigma_{0}^{2} \sum_{i=1}^{r}\left(v_{i} / \lambda_{i}\right)^{2}
$$

From the formula (11) can be seen: if the parameter estimates for the sick, $\lambda_{i} \rightarrow 0$,so $1 / \lambda_{i} \rightarrow \infty$, and the measurements $Z$ variance $\sigma_{0}^{2}$ will be enlarged, the estimated parameters will be enlarged. In this case, the parameter estimation is very unstable, the result is not reliable or even completely wrong. Therefore, we need to modify the $\lambda$, According to the multi station direction finding cross location algorithm, the eigenvalue distribution of the information matrix is continuous, we used the modified singular value decomposition (MSVD) method to correct the $\lambda$.

The MSVD method modified $\lambda_{i}$ is out of the above purpose, the value of the $\lambda_{i}$ is modified and not omited, so the number of $\lambda_{i}$ will not be reduced. There are many methods to modify the $\lambda_{i}$, and the more simple modification is $\bar{\lambda}_{i}=\lambda_{i}+\alpha / \lambda_{i}, \alpha$ is more than zero. So the modified $S^{-1}$ is

$$
S^{-1}=\operatorname{diag}\left(\frac{\lambda_{1}}{\lambda_{1}^{2}+\alpha_{1}}, \ldots, \frac{\lambda_{r}}{\lambda_{r}^{2}+\alpha_{r}}, 0, \ldots, 0\right)
$$

Written in vector form:

$$
\hat{b}_{\alpha}=\sum_{i=1}^{r} \frac{\lambda_{i}}{\lambda_{i}^{2}+\alpha_{i}}\left(Z, v_{i}\right) v_{i}
$$

The bias estimation of variance:

$$
\operatorname{var}\left(\hat{X}_{\alpha}\right)=\sigma_{0}^{2} \sum_{i=1}^{r} v_{i}^{2} /\left(\lambda_{i}+\alpha / \lambda_{i}\right)^{2}
$$

From the formula (13) and formula (14), it can be seen that the proper selection of $\alpha$ can reduce the variance of estimation and increase the stability of estimation.

\section{SimUlation VERIFICATION}

Simulation conditions: Suppose the master station is $(0,0,0.1)$, the locations of three auxiliary stations are $(20,20,0)$, $(-20,25,0)$, and $(15,20,0)$, and the units are $\mathrm{km}$. The time measurement error is $30 \mathrm{~ns}$, the station error is $10 \mathrm{~m}$, and the correlation coefficient between the time measurement errors is 0.5 , and the target height is $10 \mathrm{~km}$, and the simulation is verified in the range of $\mathrm{x}:-100 \sim 100 \mathrm{~km}, \mathrm{y}:-100 \sim 100 \mathrm{~km}$. By comparing the GDOP mesh of Fig. 2 and Fig. 3, it can be seen that the localization accuracy of the improved algorithm is significantly higher than that of the original algorithm; compared with the GDOP contour map of Fig. 4 and Fig. 5, we can see that the localization accuracy of the improved algorithm is obviously higher than that of the original algorithm, and the cross location accuracy of multi station direction finding is improved better; Fig. 6 is an improved GDOP contour map for improved algorithms. 


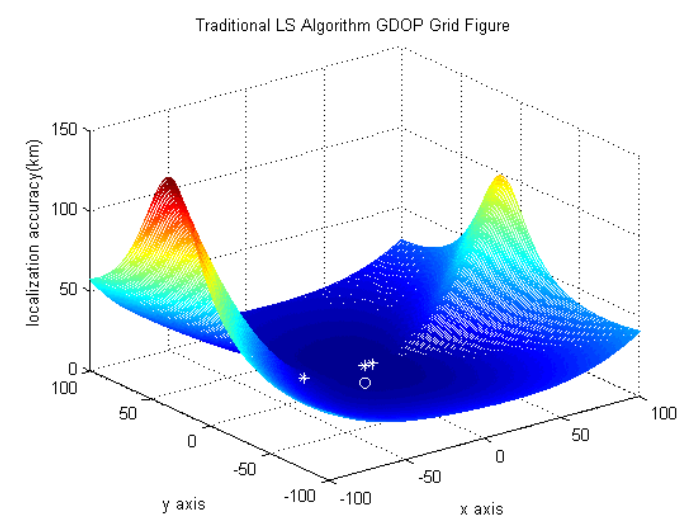

FIGURE II. TRADITIONAL LS ALGORITHM GDOP GRIDS

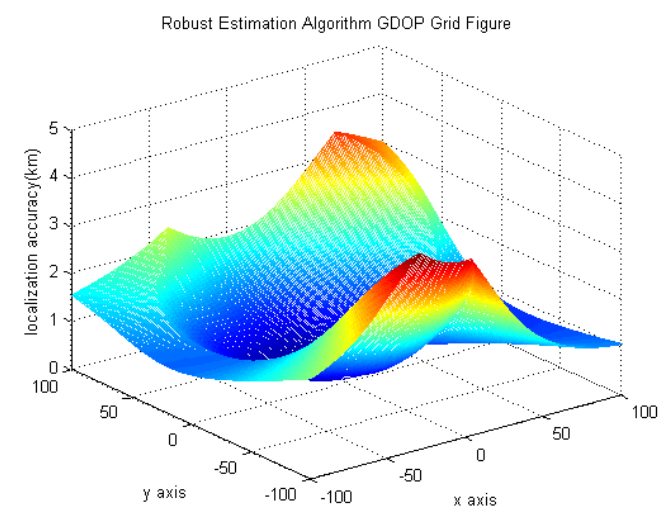

FIGURE III. ROBUST ESTIMATION ALGORITHM GDOP GRIDS

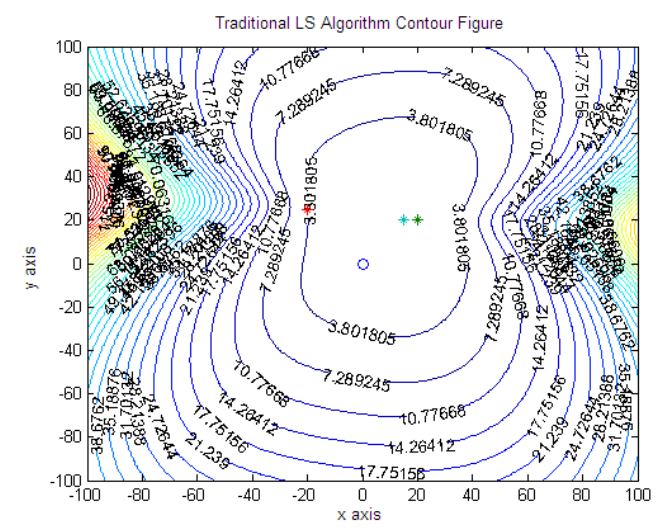

FIGURE IV. TRADITIONAL LS ALGORITHM FOR LOCATING GDOP CONTOUR LINES

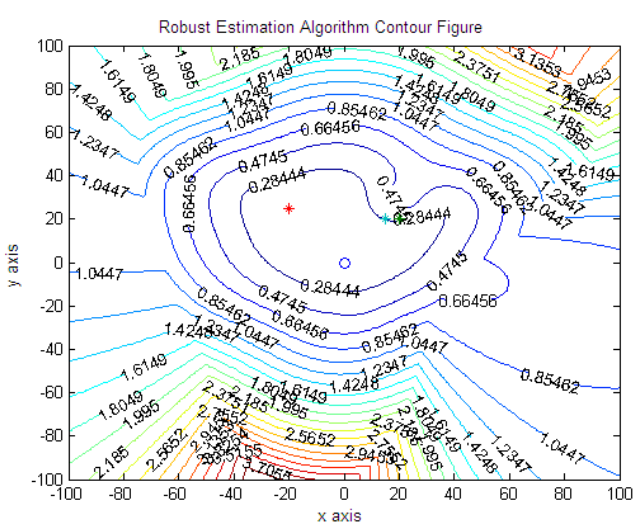

FIGURE V. ROBUST ESTIMATION ALGORITHM FOR LOCATING GDOP CONTOUR LINES

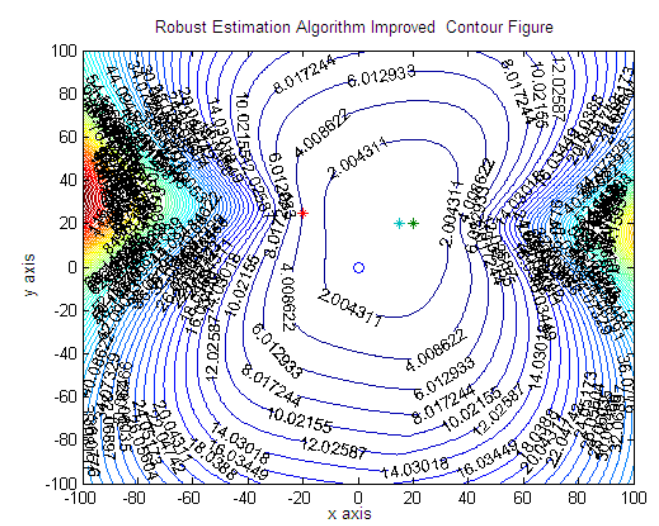

FIGURE VI. ROBUST ESTIMATION ALGORITHM TO IMPROVE THE POSITIONING OF GDOP CONTOURS

\section{CONCLUSION}

Aiming at the multi station in TDOA Location, location estimation results caused due to sensor layout unreasonable factors such as positioning equation matrix ill condition of instability, divergence and even invalid situation, we analyzes the causes of ill-condition in traditional localization algorithm Therefore, this paper puts forward a kind of stable positioning method based on singular value decomposition modification. The method doesn't require the station allocation greatly and could consider the resolution and variance assessed by positioning parameter effectively. It realizes effective inhibition to the random observation noise in the observation equation and has better engineering application value. The value simulation result indicates the positioning result is more precise and stable than traditional calculating method.

\section{REFERENCES}

[1] Chestnut, P. C. "Emitter Location Accuracy Using TDOA and Differential Doppler." IEEE Transactions on Aerospace \& Electronic Systems AES-18.2(1982):214-218.

[2] Cong, L., and W. Zhuang. "Non-line-of-sight error mitigation in TDOA mobile location." Joint Conference of the IEEE Computer and Communications Societies IEEE Xplore, 2001:659.

[3] Ho, K. C., and Y. T. Chan. "Solution and performance analysis of geolocation by TDOA." IEEE Transactions on Aerospace \& Electronic Systems 29.4(2002):1311-1322. 
[4] Catovic, A, and Sahinoglu, Z. "The Cramer-Rao bounds of hybrid TOA/RSS and TDOA/RSS location estimation schemes."Communications Letters IEEE 8.10(2004):626-628.

[5] Ho, K. C, X. Lu, and L. Kovavisaruch. "Source Localization Using TDOA and FDOA Measurements in the Presence of Receiver Location Errors: Analysis and Solution." IEEE Transactions on Signal Processing55.2(2007):684-696.

[6] Schmitz, Johannes, F. Schröder, and R. Mathar. "TDOA fingerprinting for localization in non-line-of-sight and multipath environments." Antennas and Propagation IEEE, 2016.

[7] Huang, Baoqi, L. Xie, and Z. Yang. "TDOA-Based Source Localization With Distance-Dependent Noises." IEEE Transactions on Wireless Communications 14.1(2015):468-480.

[8] Ni, Hao, G. Ren, and Y. Chang. "A TDOA location scheme in OFDM based WMANs." IEEE Transactions on Consumer Electronics54.3(2008):1017-1021.

[9] Najar, M., and J. Vidal. "Kalman tracking based on TDOA for UMTS mobile location." IEEE International Symposium on Personal, Indoor and Mobile Radio Communications IEEE, 2002:B-45-B-49 vol.1.

[10] Scheuing, Jan, and B. Yang. "Disambiguation of TDOA Estimation for Multiple Sources in Reverberant Environments." IEEE Transactions on Audio Speech \& Language Processing 16.8(2008):1479-1489.

[11] Yeredor, Arie, and E. Angel. "Joint TDOA and FDOA Estimation: A Conditional Bound and Its Use for Optimally Weighted Localization."IEEE Transactions on Signal Processing 59.4(2011):16121623.

[12] Chen xi ru, Wang song gui. Principle, method and application of regression analysis. HEFEI, Anhui Education Press.1987. 